\title{
Weakly complete domains in Grauert-type surfaces
}

\author{
Samuele Mongodi ${ }^{1}$
}

Received: 31 May 2018 / Accepted: 4 December 2018 / Published online: 11 December 2018 (c) Fondazione Annali di Matematica Pura ed Applicata and Springer-Verlag GmbH Germany, part of Springer Nature 2018

\begin{abstract}
The aim of this short note is to investigate the geometry of weakly complete subdomains of Grauert-type surfaces, i.e., open connected sets $D$, sitting inside a Grauert-type surface $X$, which admit a smooth plurisubharmonic exhaustion function. We prove that they are either modifications of Stein spaces or Grauert-type surfaces themselves, and we apply these results to the special case of Hopf surfaces.
\end{abstract}

Keywords Weakly complete · Grauert-type surfaces · Levi problem

Mathematics Subject Classification $32 \mathrm{C} 40 \cdot 32 \mathrm{E} 05 \cdot 32 \mathrm{U} 10$

\section{Introduction}

A weakly complete space is a complex space which admits a plurisubharmonic exhaustion function; if the function is strictly plurisubharmonic outside a compact set, then the space is a modification of a Stein space.

Understanding the geometry of weakly complete spaces can be thought as a generalization of the classical Levi problem; in [4], Slodkowski, Tomassini and the author classified weakly complete surfaces which admit a real analytic plurisubharmonic exhaustion function. We refer to [3] for a review of some examples and the main steps of the proof; the result, loosely stated, says that such a complex surface is either holomorphically convex or the regular level sets of the exhaustion are Levi-flat hypersurfaces foliated in dense complex curves.

These last instances are called Grauert-type surfaces.

The aim of this short note is to investigate the geometry of weakly complete subdomains of Grauert-type surfaces, i.e., open connected sets $D$, sitting inside a Grauert-type surface $X$, which admit a smooth plurisubharmonic exhaustion function.

Instances of this question can be found, for example, in the works of Miebach (see [2]) and Levenberg and Yamaguchi (see [1]), where the problem is raised for Hopf surfaces.

It turns out that a weakly complete domain in a Grauert-type surface can either be a modification of a Stein space or a Grauert-type surface itself; in particular, this implies a kind

Samuele Mongodi

samuele.mongodi@polimi.it

1 Dipartimento di Matematica, Politecnico di Milano, Via Bonardi, 9, 20133 Milan, Italy 
of stability result, namely that, if the ambient space $X$ admits a real analytic plurisubharmonic exhaustion function, then every weakly complete domain $\Omega \subseteq X$ can be endowed with a real analytic plurisubharmonic exhaustion function.

The proof we present relies on some observations that hold true not only for Grauert-type surfaces, but also for holomorphically convex ones; therefore, we present our results for any weakly complete surface with a real analytic plurisubharmonic exhaustion function.

\section{The kernel of a weakly complete space}

Let $X$ be a weakly complete complex surface, endowed with a real analytic plurisubharmonic exhaustion function $\alpha: X \rightarrow \mathbb{R}$; suppose also that $D \subseteq X$ is a weakly complete domain, with an exhaustion function $\phi: D \rightarrow \mathbb{R}$ which is plurisubharmonic and smooth.

Following [6], we denote by $\Sigma_{X}^{1}$ and $\Sigma_{D}^{1}$ their kernels; namely, $\Sigma_{X}^{1}$ is the set of points of $X$ where every plurisubharmonic exhaustion function fails to be strictly plurisubharmonic.

By [6, Lemma 3.1], we can suppose that both $\alpha$ and $\phi$ are minimal for $X$ and $D$, respectively, i.e., that $\Sigma_{X}^{1}$ is exactly the set of points where $\alpha$ is not strictly plurisubharmonic and $\Sigma_{D}^{1}$ is exactly the set of points where $\phi$ is not strictly plurisubharmonic. Moreover, by [4, Proposition 3.1], we can assume that both $\alpha$ and $\phi$ are nonnegative.

The following lemma collects some properties of kernels and minimal functions that we will need later on.

Lemma 2.1 Let $X, D, \alpha, \phi$ be as above. Then

(1) $\Sigma_{D}^{1} \subseteq \Sigma_{X}^{1}$

(2) for every $p \in \Sigma_{D}^{1}$ which is a regular point for $\phi, d \alpha(p)=\lambda_{p} d \phi(p)$

(3) for every $p \in \Sigma_{D}^{1}, d \phi(p)$ vanishes on the kernel of $\partial \bar{\partial} \phi(p)$.

Proof Take a point $p \in D$; if $p \notin \Sigma_{X}^{1}$, then the Levi form of $\alpha$ is positive definite at $p$, so $\alpha+\phi$ is a plurisubharmonic exhaustion function for $D$ which is strictly plurisubharmonic at $p$; hence, $p \notin \Sigma_{D}^{1}$. This proves (1).

Now, observe that, if $\psi$ is a nonnegative plurisubharmonic function and $q$ is a regular point for $\psi$, then

$$
\partial \bar{\partial} \psi^{2}=2(\partial \psi \wedge \bar{\partial} \psi+\psi \partial \bar{\partial} \psi)
$$

is again positive semidefinite and the kernel of the associated linear map is given by the intersection of the kernel of $\partial \bar{\partial} \phi$ and the kernel of $\partial \psi$ (or, as $\psi$ is real valued, of $d \psi$ ). Therefore, if $p \in \Sigma_{D}^{1} \subseteq \Sigma_{X}^{1}$, then both $\phi$ and $\phi^{2}$ must both have degenerate Levi forms at $p$; hence, $d \phi$ has to vanish on the kernel of $\partial \bar{\partial} \phi$. So (3) is proved.

Now, consider $\phi^{2}+\alpha^{2}$. In order for its Levi form to be degenerate at $p$, the kernels of $d \phi$ and $d \alpha$ and the kernels of $\partial \bar{\partial} \phi$ and $\partial \bar{\partial} \alpha$ must all coincide; hence, (2) follows.

From the Main Theorem in [4], in particular it follows that, for every $p \in \Sigma_{X}^{1}$, regular point for $\alpha$, there exist an irreducible complex curve $F_{p}$ and an injective holomorphic map $i_{p}: F_{p} \rightarrow X$ such that $p \in C_{p}=i\left(F_{p}\right) \subseteq \Sigma_{X}^{1} \cap\{\alpha=\alpha(p)\}$. Moreover, $T_{p} C_{p}$, if defined, is the kernel of $\partial \bar{\partial} \alpha(p)$.

Proposition 2.2 Consider $p \in \Sigma_{D}^{1}$ and suppose that $\phi(p)$ is a regular value for $\phi$. Then $C_{p} \subseteq \Sigma_{D}^{1}$ 
Proof By [6, Lemma 4.1], $\Sigma_{D}^{1} \cap\{\phi=\phi(p)\}$ is foliated in complex curves; let $S_{p}$ be the leaf passing through $p$. By Lemma 2.1-(2), as $\phi$ is constant along $S_{p}$, so it is $\alpha$. Therefore, the immersed complex curve $S_{p}$ sits in $\Sigma_{D}^{1} \cap\{\phi=\phi(p)\}$ as well as in $\Sigma_{X}^{1} \cap\{\alpha=\alpha(p)\}$. But the latter, by the Main Theorem in [4], is either union of a finite number of irreducible compact curves or it is a real three-dimensional space foliated with complex leaves, which are then the only complex curves in it. Therefore, $S_{p}=C_{p}$.

Putting these observations together with the geometric structure of $X$ given by the Main Theorem in [4], we obtain the result we claimed.

Theorem 2.3 Let $X$ be a weakly complete complex surface endowed with a real analytic plurisubharmonic exhaustion function $\alpha: X \rightarrow \mathbb{R}$; consider $D \subseteq X$ a domain with a $\mathcal{C}^{\infty}$ plurisubharmonic exhaustion function $\phi: D \rightarrow \mathbb{R}$. Then, one of the following is true:

(1) D is a modification of a Stein space

(2) both $D$ and $X$ are union of complex curves

(3) both $D$ and $X$ are Grauert-type surfaces.

Proof We separate two cases.

\section{First case}

If we find a point $p \in \Sigma_{D}^{1}$ and a minimal function $\psi$ for $D$ such that the level set

$$
Z_{\psi}(p)=\{q \in D: \psi(q)=\psi(p)\}
$$

is regular for $\psi$, then, by Proposition 2.2, $C_{p} \subseteq \Sigma_{D}^{1} \subseteq D$.

Obviously, also the closure of $C_{p}$ will be contained in $D$; in the case of Grauert-type surfaces, this implies that $D$ contains the connected component of the level set of $\alpha$ which contains $p$.

Now, if $X$ is fibered in compact curves, we have a (holomorphic) proper map with connected fibers $F: X \rightarrow T$, where $T$ is an open connected Riemann surface. If $X$ is a surface of Grauert type with $M=\min \alpha$ of real dimension 2, then there is a (pluriharmonic) proper map [4, Main Theorem] with connected fibers [5, Theorem 3.1] $F: X \backslash M \rightarrow T$, where $T$ is the real line. If $M$ has real dimension 3, up to passing to a double cover $X^{*}$, we find a (pluriharmonic) proper map with connected fibers $F: X^{*} \rightarrow T$, where $T$ is an open connected subset of the real line.

In all these three scenarios, we have a proper map with connected fibers $F: X^{*} \rightarrow T$, where $X^{*}$ is either $X, X \backslash M$ or a double cover of $X$ and $T$ is either an open connected Riemann surface or an open connected subset of the real line.

We define

$$
\Omega=\left\{x \in T: F^{-1}(x) \subseteq D\right\}
$$

and, by the first part of this proof, we know that $\Omega \neq \emptyset$; we also set $V=F(D)$. We know that $V$ is open and connected.

Consider a sequence $\left\{x_{n}\right\} \subset \Omega$, converging to a point $x \in V$. If the fibers of $F$ are irreducible compact complex curves, then obviously any plurisubharmonic function is constant on them. If the fibers of $F$ are smooth Levi-flat hypersurfaces with dense complex leaves, then again any plurisubharmonic function is constant on them (see the end of the proof of [4, Corollary 3.8]); $\alpha$ being real analytic and proper, its critical values cannot accumulate, so, up to passing to a subsequence, we can suppose that $F^{-1}\left(x_{n}\right)$ is always contained in a smooth Levi-flat hypersurface. So, in both cases, as $F^{-1}\left(x_{n}\right)$ is contained in $D$, then $\phi$ is constant on it, for every $n$. 
For every $p \in F^{-1}(x) \cap D$ (which is nonempty), there is a sequence of points $p_{n}$ such that $p_{n} \in F^{-1}\left(x_{n}\right), p_{n} \rightarrow p$. This implies that $\phi$ is constant on $F^{-1}(x) \cap D$, but then $F^{-1}(x) \subset D$, because $\phi$ is an exhaustion function and the fibers of $F$ are compact and connected. Hence, $\Omega$ is closed in $V$.

On the other hand, if $x \in \Omega$, then $\phi$ is constant on $F^{-1}(x)$, which is then a compact subset of $D$. By an easy topological argument, there exists a neighborhood $U$ of $x \in T$ such that $F^{-1}(U) \subseteq D$, i.e., $U \subseteq \Omega$, i.e., $\Omega$ is open in $V$.

Therefore, $\Omega$ is both open and closed in $V$, which is connected; so $\Omega=V$, i.e., $D=$ $F^{-1}(V)$.

\section{Second case}

Suppose that for every point $p \in \Sigma_{D}^{1}$, the level set $Z_{\phi}(p)$ is singular. By Sard's theorem, there exists a sequence $\left\{c_{n}\right\}_{n \in \mathbb{N}} \subseteq \mathbb{R}$ of regular values of $\phi$ such that $c_{n} \rightarrow+\infty$; hence, there exists a sequence of regular level sets $Z_{\phi}\left(p_{n}\right)$ with $p_{n} \rightarrow b D$.

By hypothesis, none of these level sets intersects $\Sigma_{D}^{1}$, so, $\phi$ being minimal, all these levels are strongly pseudoconvex. By a standard argument, this implies that $D$ is a modification of a Stein space.

Remark 2.1 All the contents of this section, apart from the second case in the previous theorem, hold when $\phi$ is supposed to be only $\mathcal{C}^{2}$; however, in order to apply Sard's theorem, we need $\phi$ to be at least $\mathcal{C}^{4}$.

\section{Applications}

One special case of weakly complete surfaces with a real analytic plurisubharmonic exhaustion function is Hopf surfaces with one compact curve removed (see [4, Section 2] for a worked example). Applying Theorem 2.3 to this particular case, we obtain a different proof for the Main Theorem in [2] and Theorem 1.1. in [1], under the hypothesis of the existence of a smooth plurisubharmonic exhaustion.

More generally, suppose $X$ is the total space of a topologically trivial line bundle over a compact Riemann surface $M$; if the class in $H^{1}\left(M, \mathcal{S}^{1}\right)$ associated with the bundle is unipotent, then $X$ is fibered in compact curves; otherwise, it is a Grauert-type surface (see [4, Section 2] and [5, Section 4] for related discussions and examples).

In both cases, we have a minimal plurisubharmonic function whose only critical level is the minimum level, coinciding with $M$; so, any open set containing $M$ will also contain a regular level set of the exhaustion function.

Combining these observations with Theorem 2.3, we obtain the following

Corollary 3.1 Suppose $X$ is the total space of a topologically trivial line bundle $\xi$ over a compact Riemann surface $M$ and let $D \subset X$ be a weakly complete domain with a smooth plurisubharmonic exhaustion. Then one of the following happens:

(1) D is Stein

(2) both $D$ and $X$ are fibered in complex curves

(3) both $D$ and $X$ are Grauert-type surfaces.

Proof From Theorem 2.3 we know that, if cases (2) and (3) don't apply, then $D$ is a modification of a Stein space. However, if $D$ were not Stein, then it would contain some compact curves. If $X$ is a surface of Grauert type, the only compact curve in it is $M$, which doesn't have any Stein neighborhood; on the other hand, if $X$ is fibered in complex curves, as soon 
as $D$ contains one of them, it is a union of curves, repeating the argument in the end of the first case in the proof of Theorem 2.3.

Therefore, $D$ is Stein.

Remark 3.1 The proof we have just given applies whenever $X$ is a weakly complete surface which admits a real analytic plurisubharmonic exhaustion function whose only critical level set is the minimum level set.

\section{References}

1. Levenberg, N., Yamaguchi, H.: Pseudoconvex domains in the Hopf surface. J. Math. Soc. Jpn. 67(1), 231-273 (2015). https://doi.org/10.2969/jmsj/06710231

2. Miebach, C.: Pseudoconvex non-Stein domains in primary Hopf surfaces. Izv. Ross. Akad. Nauk Ser. Mat. 78(5), 191-200 (2014). https://doi.org/10.1070/IM2014v078n05ABEH002717

3. Mongodi, S., Slodkowski, Z., Tomassini, G.: On weakly complete surfaces. C. R. Math. 353(11), 969-972 (2015). https://doi.org/10.1016/j.crma.2015.08.009

4. Mongodi, S., Słodkowski, Z., Tomassini, G.: Weakly complete complex surfaces. Indiana Univ. Math. J. 67(2), 899-935 (2018). https://doi.org/10.1512/iumj.2018.67.6306

5. Mongodi, S., Slodkowski, Z., Tomassini, G.: Some properties of Grauert type surfaces. Int. J. Math. 28(8), 1750063 (2017). https://doi.org/10.1142/S0129167X1750063X

6. Slodkowski, Z., Tomassini, G.: Minimal kernels of weakly complete spaces. J. Funct. Anal. 210(1), 125147 (2004). https://doi.org/10.1016/S0022-1236(03)00182-4 